appreciation of my encouraging self-reflection and understanding, and the observable values in my carrying out of the assessment, which could be compared to those of other assessors.

Conclusion. WPBAs have their merits, and shortfalls. I am aware of my values and beliefs when utilising them, and have identified a plan to further develop my own practice. This case study is particular, but possibly not unique, in how WPBAs are used in medical education.

\section{SW Neuronet - neuroscience for psychiatrists update day}

Gabrielle Churchhouse ${ }^{1 \star}$, Lindsey Sinclair ${ }^{2}$ and Simon George Morris ${ }^{3}$

${ }^{1}$ Gloucestershire Health and Care NHS Foundation Trust; ${ }^{2}$ University of Bristol, Avon and Wiltshire Mental Health Partnership NHS Trust and ${ }^{3}$ Avon and Wiltshire Mental Health Partnership NHS Trust ${ }^{\star}$ Corresponding author.

doi: 10.1192/bjo.2021.375

Aims. The Royal College of Psychiatrists Neuroscience Project was established to promote greater integration of modern neuroscience into psychiatric training and practice. Regional "Neuronets" are being established to develop local learning opportunities. As the Southwest Neuronet, we sought to establish a high quality and sustainable regional educational event promoting modern neuroscience in psychiatry.

Method. We developed and ran two events in collaboration with the Neuroscience Project, a whole day in-person event in September 2019 and a half day online event in January 2021. Attendees were invited from the Southwest with the latter event being shared more widely through other "Neuronets". Both featured talks by leading experts in the neuroscience of psychiatry. The first was themed around "Neuroscience from the lab to the clinic", building on basic research methodologies to their applications in clinical psychiatry. Our pandemic era online event, "Neuroscience of psychosis", was structured around an evolving clinical case. Both featured interactive elements using audience polling technology to gather views and collate questions. Feedback was gathered through an online survey with individual session ratings and event ratings.

Result. 154 people attended the in-person event from across the South West Division. This included psychiatry trainees, consultants and a small number of other mental health professionals. 382 people signed up to our online event with 262 attending live and others watching recorded sessions. Feedback response rates were $42 \%$ and $33 \%$ respectively. Feedback on the practical arrangements was highly positive, particularly highlighting pre-event communication. Attendees valued the high calibre of speakers and particularly rated topics of psychiatric genetics, novel antidepressants, and autoimmune psychosis. Environmental sustainability was a prominent theme in our first event with support for our paperless approach but highlighted further potential to reduce waste associated with catering. Overall, attendees valued the opportunity to build on knowledge of basic research techniques but also wished to see greater focus on clinical applications of neuroscience, which we had responded to with the inclusion of a clinical case to frame our online event.

Conclusion. These events provide a prototype for low-cost regional neuroscience in psychiatry education events, in-person or online. Sustainability in terms of cost, human resources for organisation, and environmental impact are all important considerations for such events. We plan to continue to run these annually, forming part of the legacy of the Neuroscience Project. In line with feedback received, we seek to maximise the clinical relevance but also share novel research techniques encountered in the literature.

\section{Evaluating adult forensic staff knowledge of} olanzapine long-acting injection post injection syndrome: a service improvement project

Annalie Clark*, Onagh Boyle, Suhanthini Farrell and Catrin Evans

Greater Manchester Mental Health NHS Foundation Trust ${ }^{\star}$ Corresponding author.

doi: 10.1192/bjo.2021.376

Aims. Post injection syndrome (PIS) is a serious complication that can occur after Olanzapine Long Acting Injection (LAI). It can occur without any derangement in physical observations. It is important that patients are monitored appropriately following administration of Olanzapine LAI to ensure that symptoms of PIS are appropriately identified and managed. This project aimed to evaluate the current level of knowledge about PIS in two staff groups within an Adult Forensic Service - in-patient nursing staff and junior doctors and advanced practitioners (APs) providing medical cover to inpatient wards.

Method. Electronic surveys evaluating knowledge about the symptoms of PIS, monitoring requirements and management of possible PIS were circulated to inpatient nursing staff, junior doctors and APs working within an Adult Forensic Service in the North West of England.

Result. 1) Nursing staff knowledge - 26 nursing staff completed the survey. $4.5 \%$ of nurses correctly identified all symptoms of PIS and $72.7 \%$ believed that tachycardia or hypotension occur in PIS. $22.7 \%$ of nurses identified the correct management plan if a patient feels unwell following Olanzapine LAI. 40.9\% would only request a medical review if physical observations were abnormal. 2) Junior doctor and AP knowledge - 6 doctors and 6 advanced practitioners completed the survey. $17 \%$ of doctors and APs correctly identified all symptoms of PIS. 50\% believed hypotension or tachycardia were symptoms of PIS. $25 \%$ of doctors and APs identified correct management of PIS and $16.7 \%$ believed that the patient should be managed on the psychiatric ward unless physical observations became abnormal.

Conclusion. Levels of knowledge about the symptoms and management of PIS are low within this Adult Forensic Service. Knowledge of PIS and management of suspected PIS needs to be improved in nursing staff, junior doctors and advanced practitioners to ensure correct identification and safe management. In response to these findings, a care plan for monitoring of patients after Olanzapine LAI was developed. This included a structured monitoring proforma for completion post depot administration and instructions for managing suspected PIS. This care plan is kept in the front of the drug chart of all patients prescribed Olanzapine LAI. One-page educational summaries on PIS were written and circulated to nursing staff, junior doctors and APs. Information on Olanzapine LAI use and PIS were included in junior doctor induction materials and on-call handbook, to improve trainee awareness and knowledge.

\section{Supporting general adult psychiatry higher trainees to develop research competencies: a training improvement project}

Annalie Clark ${ }^{1 \star}$, John Stevens ${ }^{2}$ and Sarah Abd El Sayed ${ }^{3}$

${ }^{1}$ Greater Manchester Mental Health NHS Foundation Trust; ${ }^{2}$ Mersey Care NHS Foundation Trust and ${ }^{3}$ Greater Manchester Mental Health NHS Foundation Trust ${ }^{\star}$ Corresponding author.

doi: 10.1192/bjo.2021.377 